\title{
The EGFR Inhibitor Gefitinib Enhanced the Response of Human Oral Squamous Cell Carcinoma to Cisplatin In Vitro
}

\author{
Ashraf Khalil ${ }^{1,2} \cdot$ Mark J. Jameson ${ }^{1}$
}

Published online: 21 August 2017

(c) The Author(s) 2017. This article is an open access publication

\begin{abstract}
Introduction The epidermal growth factor receptor (EGFR) is highly expressed in a variety of solid tumors including oral cavity squamous cell carcinoma (OSCC) and has been implicated in the resistance of these tumors to cisplatin. This study was performed to determine if the EGFR tyrosine kinase inhibitor gefitinib could enhance the cytotoxic effect of cisplatin on OSCC cells in vitro.

Methods The expression of EGFR and the phosphorylation of its downstream signaling to ERK, and AKT pathway were detected by Western blotting. Cell proliferation and survival were determined by AlamarBlue and colony formation assay respectively. Cells apoptosis were determined by Western blotting for cleaved PARP protein and by flowcytometry of cells stained with Annexin V and PI.

Results Cal27, OSC19, and SCC25 cells treated with gefitinib $1 \mu \mathrm{M}$ demonstrated reduced phosphorylation of
\end{abstract}

Ashraf Khalil

ashkali12010@gmail.com

$\bowtie$ Mark J. Jameson

mark.jameson@virginia.edu

1 Division of Head and Neck Oncologic and Microvascular Surgery, Department of Otolaryngology-Head and Neck Surgery, University of Virginia Health System, Charlottesville, VA, USA

2 Department of Biochemistry, National Liver Institute, Menoufiya University, Shibin Al Kawm, Egypt
EGFR, AKT, and ERK proteins with very limited inhibition of proliferation. Cisplatin inhibited proliferation of the same cell lines in a dose-dependent manner. The concentration producing $50 \%$ inhibition $\left(\mathrm{IC}_{50}\right)$ for cisplatin decreased in the presence of gefitinib $1 \mu \mathrm{M}$, and a combination of cisplatin $5 \mu \mathrm{M}$ and gefitinib $1 \mu \mathrm{M}$ caused synergistic growth inhibition and synergistic reduction in cell survival. The growth inhibitory effect of the combination was associated with reduced ERK and AKT activation, increased poly ADP ribose polymerase (PARP) cleavage, and increased apoptosis.

Conclusion Thus, in OSCC cells in vitro, inhibition of EGFR activity with gefitinib enhances the apoptotic effect of cisplatin. This has potential implications for enhancement of cisplatin effectiveness in tumors that over-express the EGFR.

\section{Key Points}

Oral cavity squamous cell carcinoma (OSCC) cell lines Ca127, OSC19, and SCC25 express epidermal growth factor receptor (EGFR) at high levels with low basal phosphorylated EGFR (pEGFR).

OSCC cell lines have functional EGFR-ERK and EGFR-AKT signaling pathways. At $1 \mu \mathrm{M}$, gefitinib reduces AKT and ERK activation in unstimulated and EGF-stimulated cells.

Cisplatin inhibits OSCC cell growth, proliferation, and survival in a dose-dependent manner.

Combination of cisplatin with gefitinib enhances the cytotoxicity of cisplatin. This is associated with increased poly ADP ribose polymerase (PARP) cleavage and increased apoptotic cell populations. 


\section{Introduction}

The epidermal growth factor receptor (EGFR) has been implicated in the survival and proliferation of cancer cells. EGFR is highly expressed in human oral cavity squamous cell carcinomas (OSCCs). High EGFR expression has been associated with resistance to chemotherapeutic agents used in the treatment of OSCCs such as cisplatin, 5-fluorouracil (5FU), cyclophosphamide, and doxorubicin [1-3].

Via downstream signaling through extracellular signalregulated kinase (ERK) and AKT, the EGFR is implicated in multiple aspects of cancer cell physiology, including survival, proliferation, invasion, metastasis, angiogenesis, and apoptosis [4-6]. EGFR has already been recognized as a therapeutic target in head and neck squamous carcinomas, and a variety of EGFR inhibitors are currently used in the treatment of several human cancers [7-11].

Gefitinib is a low molecular weight tyrosine kinase inhibitor [12] that competes for ATP binding to the catalytic kinase domain of EGFR, thus inhibiting phosphorylation of EGFR and its downstream signaling pathways. Preclinical in vitro studies showed that EGFR inhibition with gefitinib results in decreased cell proliferation, survival, and migration with sensitivity to the drug (concentration producing $50 \%$ inhibition $\left[\mathrm{IC}_{50}\right]$ ranged from $<1$ to $13 \mu \mathrm{M})$ depending on the cancer cell type and the presence or absence of a sensitizing mutation in the EGFR protein [13]. Early clinical trials showed that gefitinib is generally well tolerated in patients with a wide range of solid tumor types including lung, head and neck, colon, breast, and prostate cancers [14-16]. Since the introduction of tyrosine kinase inhibitors (TKIs) in clinical use for solid tumors in 2003, several molecular biomarkers, including gene mutations, EGFR protein expression, and EGFR gene copy number, have been identified and suggested to have potential value in predicting responses to TKI treatment [17-21].

Cisplatin is a chemotherapeutic cytotoxic DNA-damaging alkylating drug used in the treatment of various solid tumors, often in combination with other chemotherapeutic agents. In addition to playing a key role in the therapy of many other cancers, cisplatin is a crucial component in the treatment of head and neck cancers, including OSCC [20, 22]. Intrinsic and acquired drug resistance is a major drawback of cisplatin in clinical use. The molecular mechanisms of cisplatin resistance remain indistinct, but increased expression and activation of EGFR signaling pathways is associated with decreased cellular sensitivity to cisplatin. It has been noted that EGFR inhibitors can overcome some cisplatin insensitivity in EGFR overexpressing cancers [23-25]. In animal models and in in vitro studies, the combination of an anti-EGFR monoclonal antibody with cisplatin has shown synergism in inhibiting cell proliferation and inducing apoptosis in some cisplatinresistant OSCC cell lines [26].

In the present study, we evaluate the effect of combining the EGFR-TKI gefitinib with cisplatin on in vitro proliferation, survival, cellular signaling, and apoptosis of OSCC cell lines Cal27, OSC19, and SCC25. These cell lines are known to express wild-type EGFR and have intact downstream ERK and AKT signaling pathways, which make them suitable models to study the effects of EGF-TKIs in OSCC.

\section{Materials and Methods}

\subsection{Tissue Culture and Reagents}

Cal27 and SCC25 cells were obtained from American Type Culture Collection. OSC19 cells were kindly provided by Dr. Jeffrey Myers (The University of Texas MD Anderson Cancer Center, Houston, TX, USA). Cells were grown in Dulbecco's Modified Eagle's Medium/F12 supplemented with 5\% fetal bovine serum (FBS) and 1\% penicillin/ streptomycin at $37{ }^{\circ} \mathrm{C}$ and $5 \% \mathrm{CO}_{2}$. As extended in vitro cell culture and clonal expansion can lead to the emergence of new genotypes and altered cellular phenotypes over time, all experiments were performed on early passage cells between passage number 3 and 10. Chemical reagents were obtained as follows: cisplatin (purity $>99.9 \%$ ), crystal violet, and sodium orthovanadate from Sigma-Aldrich (St. Louis, MO, USA); gefitinib (purity $>99 \%$ ) from AstraZeneca (Macclesfield, UK); recombinant human epidermal growth factor (rhEGF) from Cell Sciences (Canton, MA, USA); alamarBlue and trypan blue from Invitrogen (Carlsbad, CA, USA); anti-EGFR, anti-phosphorylated EGFR (pEGFR), anti-ERK1/2, anti-pERK1/2, anti-poly ADP ribose polymerase (PARP), anti- $\beta$-actin, and anti$\gamma \mathrm{H} 2 \mathrm{AX}$ from Cell Signaling (Beverly, MA, USA); annexin V-fluorescein isothiocyanate (FITC) and propidium iodide (PI) from Millipore (Bedford, MA, USA); and dimethylsulfoxide (DMSO), dithiothreitol (DTT), FBS and methanol from Thermo Fisher Scientific (Waltham, MA, USA).

\subsection{Immunoblot}

Treated cells in 6-cm dishes were washed with ice-cold phosphate-buffered saline (PBS) containing sodium orthovanadate $2 \mathrm{mM}$ (to preserve the protein tyrosyl phosphorylation state in cells and cells lysates) [25], collected, resuspended in lysis buffer, and processed as previously described $[15,16]$. Briefly, lysates were vortexed, incubated at $4{ }^{\circ} \mathrm{C}$ for $30 \mathrm{~min}$, and centrifuged at $13,000 \mathrm{rpm}$ 
for 15 min at $4{ }^{\circ} \mathrm{C}$. The supernatant was collected and sample buffer containing DTT $0.1 \mathrm{M}$ was added. Proteins were resolved by SDS-PAGE on a $12 \%$ polyacrylamide gel and then electrophoretically transferred to a polyvinyllidene fluoride (PVDF) membrane (Millipore) using a semidry transfer device (Trans-Blot, Bio-Rad, Hercules, CA, USA). Membranes were subsequently blocked with casein $1 \%$ in PBS buffer (Bio-Rad, Hercules, CA, USA) for $2 \mathrm{~h}$ at room temperature, incubated with the appropriate primary antibody at 1:1000 dilution overnight at $4{ }^{\circ} \mathrm{C}$. Membranes were incubated with infrared-labeled secondary antibodies (anti-mouse 680 Alexa, Molecular Probes, Eugene, OR, USA) or anti-rabbit IRDYE 800 (Rockland Immunochemicals, Gilbertsville, PA, USA) at 1:15,000 dilutions for $2 \mathrm{~h}$ at room temperature. Specific protein bands were detected and quantified using Odyssey Infrared Imaging System and Software version 1.2 from Li-Cor Biosciences (Lincoln, NE, USA).

\section{3 alamarBlue Cell Proliferation Assay}

Cells were plated at 5000 cells per well in a 96-well plate, grown for $24 \mathrm{~h}$, and treated with cisplatin and/or gefitinib dissolved in DMSO added directly into the media. Because previous studies had shown that metabolic hydrolysis of cisplatin could occur within $5 \mathrm{~min}$, cisplatin was added after gefitinib [27]. Control untreated cells were treated with an equal concentration of DMSO that did not exceed $0.1 \%$; DMSO alone at $0.1 \%$ had no effect. All conditions were assessed in triplicate. Plates were incubated for $72 \mathrm{~h}$ at $37^{\circ} \mathrm{C}$. alamarBlue was added to each well according to the manufacturer's protocol. Cells were incubated for $3 \mathrm{~h}$ at $37{ }^{\circ} \mathrm{C}$ and the fluorescence at $540 \mathrm{~nm}$ was recorded. Data were expressed as means \pm standard error of the mean (SEM).

\subsection{Colony Formation Assay}

Cal27 and OSC19 cells were seeded at 250 cells per well in 6-well plates and incubated overnight at $37^{\circ} \mathrm{C}$. Cells were treated in triplicate with gefitinib $1 \mu \mathrm{M}$ and/or cisplatin $5 \mu \mathrm{M}$. Plates were incubated for 14 days; during this period the medium was changed twice weekly with the appropriate concentration of each drug. Plates were washed with ice-cold PBS. Colonies were then fixed with methanol for 15 min, stained with $2 \%$ crystal violet and counted. Colonies consisting of $\geq 50$ cells were scored.

\subsection{Flow Cytometry}

Cal27, OSC19, and SCC25 cells were grown in 6-cm dishes to $70 \%$ confluence in 5\% FBS. Cells were incubated with the desired concentration of cisplatin and/or gefitinib for $72 \mathrm{~h}$. The conditioned medium from each well was collected; cell monolayers were trypsinized, resuspended in the corresponding conditioned medium, centrifuged at $3000 \mathrm{rpm}$ for $3 \mathrm{~min}$ at $4{ }^{\circ} \mathrm{C}$, washed once with cold PBS, and resuspended in annexin V-FITC and PI according to the manufacturer's recommendations (Millipore). Flow cytometry was conducted in the UVA Flow Cytometry Core Facility with marker combinations as follows: cells that are viable are both annexin V and PI negative; cells in early apoptosis are annexin $\mathrm{V}$ positive and PI negative; cells in late apoptosis are annexin $\mathrm{V}$ and PI positive; necrotic/dead cells are annexin V negative and PI positive.

\subsection{Statistics}

All statistical analyses were performed using Prism 3 (GraphPad Software, San Diego, CA, USA). Comparisons of conditions were assessed using a two-tailed Student's $t$ test with results considered statistically significant when $p<0.05$.

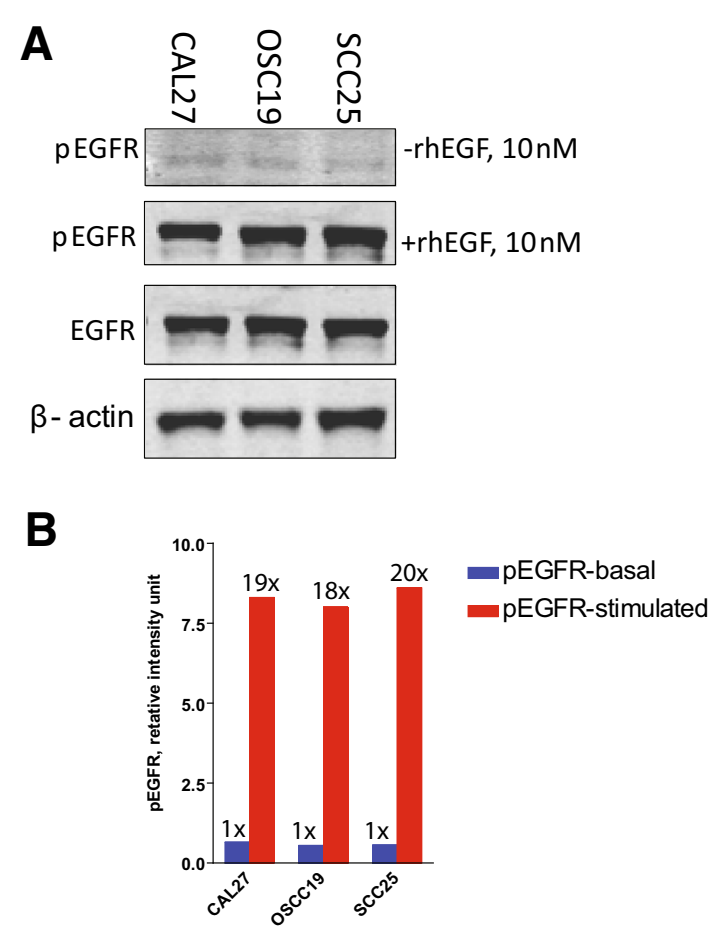

Fig. 1 EGFR and pEGFR levels in OSCC cells lines. a Immunoblot of EGFR and phosphorylated EGFR in Cal27, OSC19, and SCC25 cells lines. Cells in sub-confluent $6-\mathrm{cm}$ dishes were untreated or stimulated with rhEGF $10 \mathrm{nM}$ for $15 \mathrm{~min}$ before harvesting. $\beta$-Actin was used as a loading control. Immuno-complexes were visualized and the band intensities were quantified using Licor Odyssey System. b Densitometric analysis of immunoblot presented in graph form. Data points, pEGFR. Fold $(x)$ denotes changes in pEGFR levels compared with unstimulated normalized to $\beta$-actin protein levels. $E G F R$ epidermal growth factor receptor, $O S C C$ oral cavity squamous cell carcinoma, $p E G F R$ phosphorylated EGFR, rhEGF recombinant human epidermal growth factor 


\section{Results}

\subsection{EGFR and pEGFR Levels in OSCC Cell Lines}

EGFR and pEGFR levels in Cal27, OSC19 and SCC25 cells were characterized by immunoblot analysis of cell lysates obtained from subconfluent dishes either unstimulated or after stimulation with rhEGF $10 \mathrm{nM}$. The three cell lines express high levels of EGFR with low basal pEGFR (Fig. 1a); EGFR phosphorylation increase by 20-fold after treatment with rhEGF (Fig. 1b).

\subsection{Gefitinib Inhibits EGFR Activation and Signaling in OSCC Cells}

In Ca127, OSC19, and SCC25 cells, stimulation of EGFR with rhEGF $10 \mathrm{nM}$ resulted in increased EGFR, ERK, and AKT phosphorylation; ERK activation was more prolonged than AKT activation. One hour after treatment with gefitinib $0.25,0.5$, or $1 \mu \mathrm{M}$, there was dose-dependent inhibition of EGF-stimulated EGFR, ERK, and AKT phosphorylation (Fig. 2a). EGFR phosphorylation was completely abolished by gefitinib $0.5 \mu \mathrm{M}$, which also
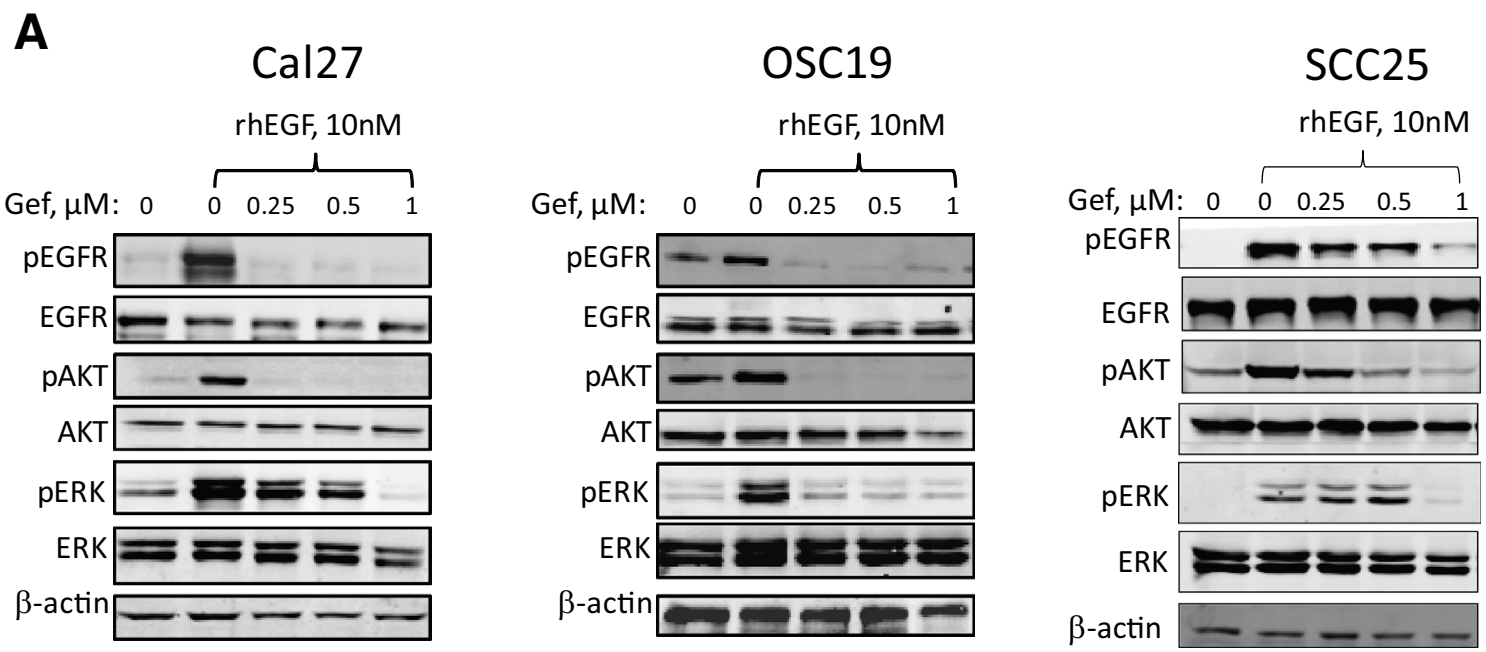

B

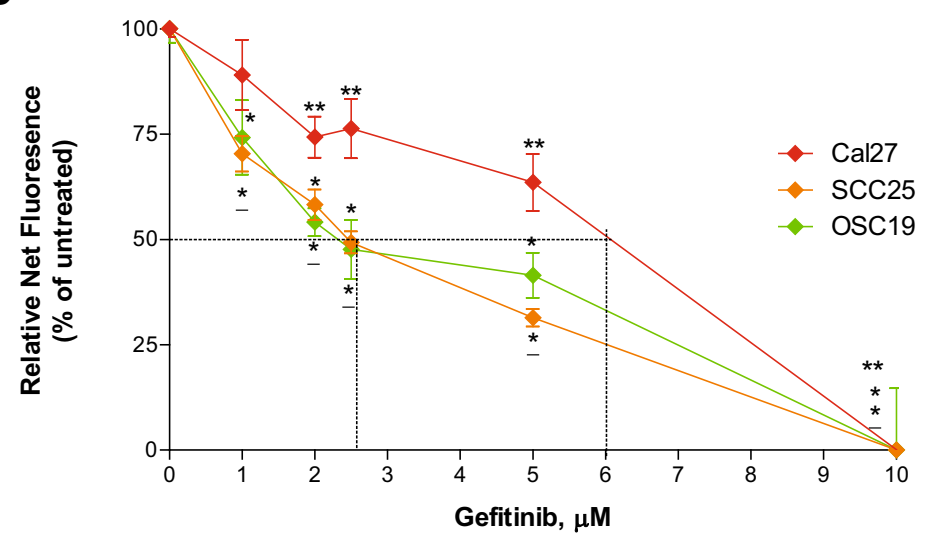

Fig. 2 Effect of gefitinib on OSCC cells signaling and growth. a Cal27, OSC19, and SCC25 cells were treated with gefitinib 0.25, 0.5 , or $1 \mu \mathrm{M}$ for $1 \mathrm{~h}$, then rhEGF $10 \mathrm{nM}$ for $10 \mathrm{~min}$. Total cellular protein was isolated and immunoblot performed using the antibodies shown. $\beta$-Actin was used to indicate equal protein loading. b Cal27, OSC19, and SCC25 were grown in 96-well plates and treated with gefitinib for $72 \mathrm{~h}$ with the concentrations shown. Proliferation was measured using the alamarBlue assay, with relative net fluorescence used as a surrogate for cell number. Relative net fluorescence is expressed as a percentage of untreated cells. Values represent means \pm standard error of the mean (SEM) of three independent triplicate experiments. ${ }^{*} p<0.05$ compared with control. EGFR epidermal growth factor receptor, Gef gefitinib, $O S C C$ oral cavity squamous cell carcinoma, $p E G F R$ phosphorylated EGFR, rhEGF recombinant human epidermal growth factor 
suppressed the downstream phosphorylation of AKT; this concentration, however, did not eliminate ERK phosphorylation. Gefitinib $1 \mu \mathrm{M}$ was required to eliminate EGFRstimulated ERK activation. At this concentration, basal pERK was also eliminated, suggesting that the activation of ERK in unstimulated cells is a result of basal EGFR activation.

\subsection{Gefitinib Inhibits OSCC Cell Proliferation}

Cal27, SCC25, and OSC19 cells were treated with increasing concentrations of gefitinib and proliferation was assessed using the alamarBlue assay (Fig. 2b). Gefitinib exhibited a dose-dependent inhibition of proliferation. Cal27 was more resistant to the growth inhibitory effect of

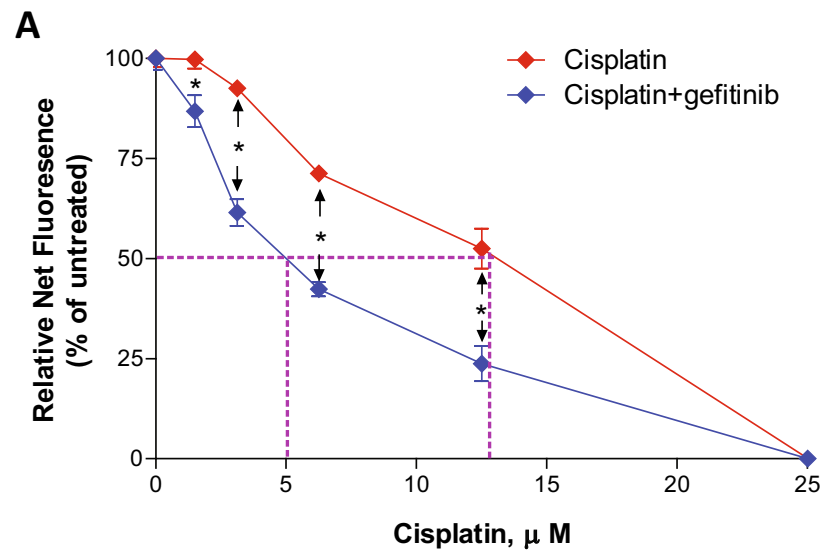

\section{B}

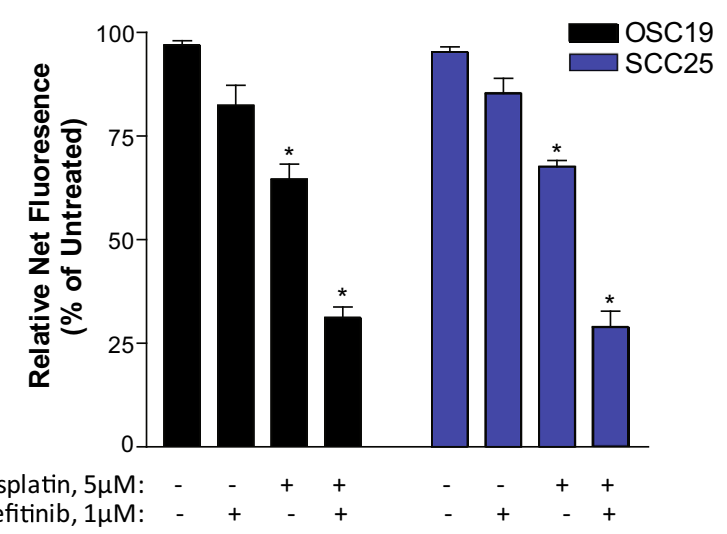

Fig. 3 Effect of cisplatin on OSCC cell growth. a Cal27 cells were grown in 96-well plates and treated with cisplatin 1.5, 3.5, 6.25, or $12.5 \mu \mathrm{M}$ for $72 \mathrm{~h}$ with or without $1 \mathrm{~h}$ of pre-treatment with gefitinib $1 \mu \mathrm{M}$ as indicated. Proliferation was measured using the alamarBlue assay. ${ }^{*} p<0.05$ when comparing cells treated with cisplatin plus gefitinib to cisplatin alone. b OSCC19 and SCC 25 cells were grown in 96-well plates and treated with cisplatin $5 \mu \mathrm{M}$ for $72 \mathrm{~h}$ in the absence or presence of gefitinib $1 \mu \mathrm{M}$. Proliferation was measured using the alamarBlue assay. Relative net fluorescence is expressed as a percentage of untreated cells. Values represent means \pm standard error of the mean (SEM) of three independent triplicate experiments. $* p<0.05$ compared with control. OSCC oral cavity squamous cell carcinoma gefitinib than OSC19 and SCC25. Gefitinib $1 \mu \mathrm{M}$ resulted in only $10 \%$ growth inhibition of Cal27 cells but 25 and $30 \%$ growth inhibition of OSC19 and SCC25 cells, respectively. The $\mathrm{IC}_{50}$ of gefitinib was $6 \mu \mathrm{M}$ for $\mathrm{Cal} 27$ cells and $2.5 \mu \mathrm{M}$ for OSC19 and SCC25 cells. Complete growth inhibition was noted at $10 \mu \mathrm{M}$ in all cell lines.

\subsection{Gefitinib Enhances Growth Inhibitory Effect of Cisplatin on OSCC Cells}

Cal27 cells were treated with cisplatin 1.5, 3.5, 6.5, 12.5, and $25 \mu \mathrm{M}$ in the absence and presence of gefitinib $1 \mu \mathrm{M}$ (Fig. 3a). Cell proliferation was determined at $72 \mathrm{~h}$ using alamarBlue. Treatment with cisplatin alone caused a dosedependent reduction in cell number with an $\mathrm{IC}_{50}$ of $12.5 \mu \mathrm{M}$. Combination with gefitinib resulted in enhanced growth inhibition by cisplatin with an $\mathrm{IC}_{50}$ of $5 \mu \mathrm{M}$. At each concentration, there was a significant difference between the growth inhibition caused by cisplatin/gefitinib combination treatment compared with cisplatin alone $(p<0.05)$.

SCC25 and OSC19 cells were treated with cisplatin $5 \mu \mathrm{M}$ and/or gefitinib $1 \mu \mathrm{M}$ (Fig. 3b). Cell proliferation was determined at $72 \mathrm{~h}$ using alamarBlue. Gefitinib alone reduced cell number by approximately $20 \%$, which was not

A

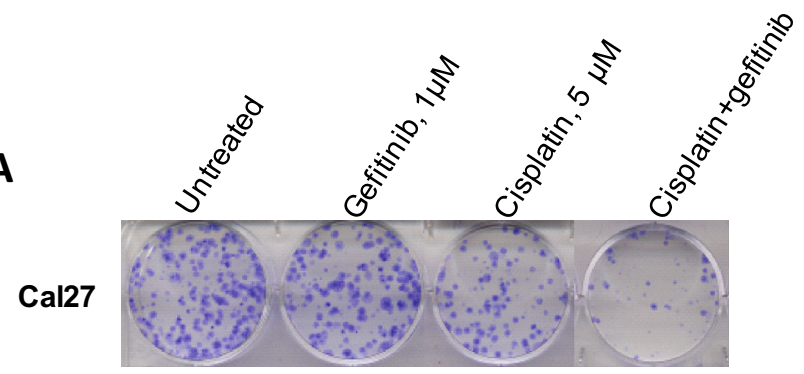

B

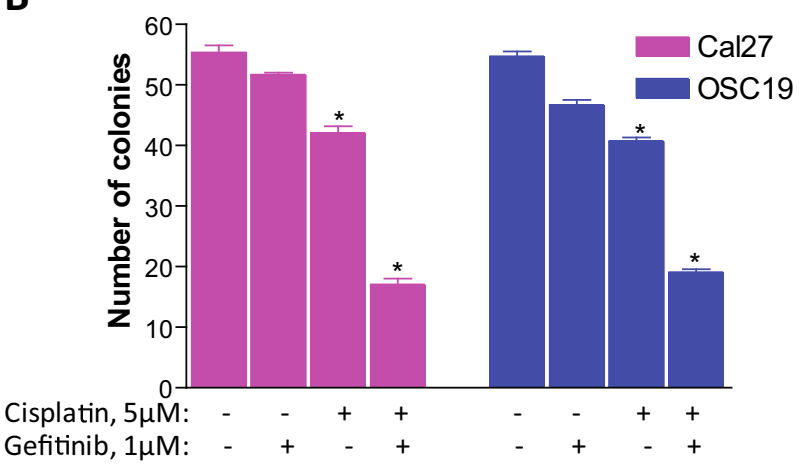

Fig. 4 Cal27 and OSCC19 colony formation after cisplatin and gefitinib treatment. Colony formation assay was performed in triplicate as described in the Methods section. a Cal27 representative stained colonies. b Mean colonies \pm standard error of the mean (SEM) at each treatment. ${ }^{*} p<0.05$ compared with control untreated cells 
statistically significant, and cisplatin alone reduced cell number by approximately $30 \%(p<0.05)$. The combination of cisplatin and gefitinib resulted in a $70-75 \%$ reduction in cell number $(p<0.05)$ indicating a synergistic growth inhibitory effect.

The EGFR expression level was very similar in the three cell lines studied (Fig. 1), thus no correlation could be identified between EGFR expression level and response to gefitinib.

\subsection{Gefitinib Enhances Cisplatin Inhibition of Clonogenic Survival of OSCC Cells}

The effect of chronic exposure (14 days) to gefitinib $1 \mu \mathrm{M}$ and/or cisplatin $5 \mu \mathrm{M}$ on clonogenic survival was evaluated using a colony formation assay (Fig. 4). Gefitinib $1 \mu \mathrm{M}$ caused a small reduction in the number of colonies formed; this was not statistically significant. Cisplatin

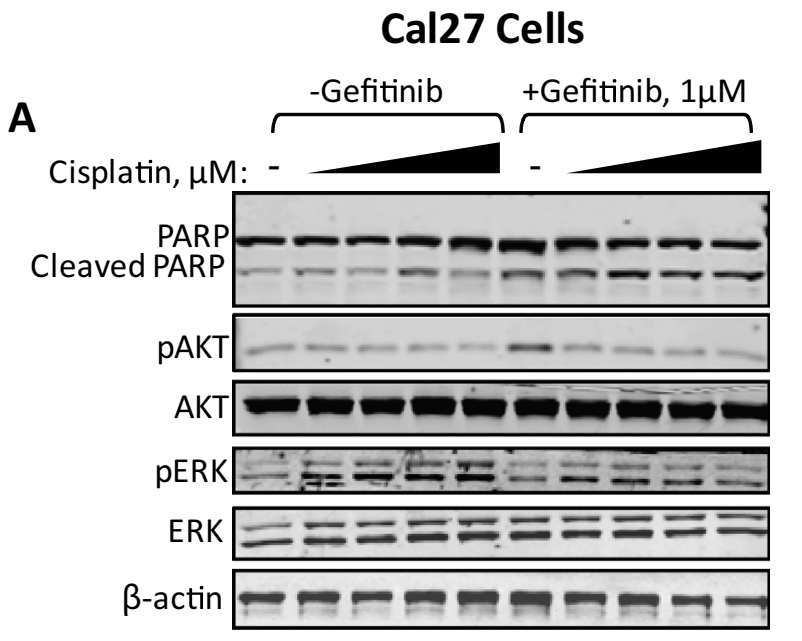

B

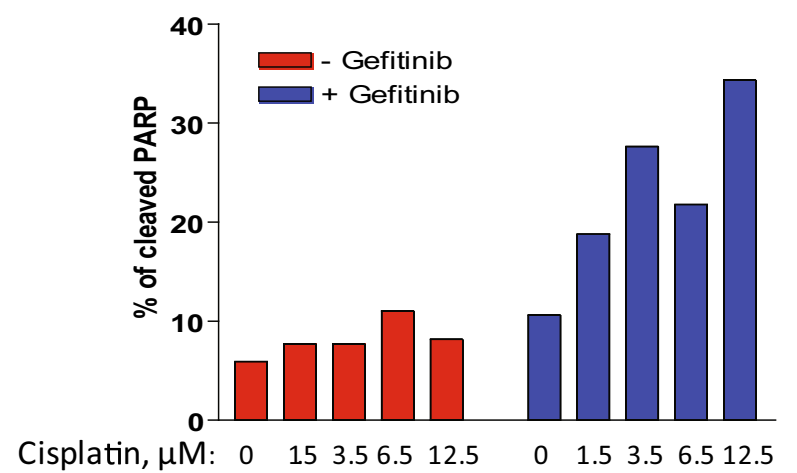

Fig. 5 Impact of cisplatin and gefitinib co-treatment on signaling in OSCC. Cal27 (a) and SCC25 (c) cells were pretreated with gefitinib for $1 \mathrm{~h}$ and then with cisplatin for $24 \mathrm{~h}$ as indicated. Both adherent and non-adherent cells were collected and subjected to immunoblot
$5 \mu \mathrm{M}$ resulted in a $20 \%$ reduction in colony formation compared with untreated cells $(p<0.05)$. Treatment with combined cisplatin and gefitinib resulted in a $65 \%$ reduction in colony formation $(p<0.05)$, again indicating a synergistic effect.

\subsection{Signaling Effects of Combined Treatment with Cisplatin and Gefitinib in OSCC Cells}

Cal27 cells either untreated or pretreated with gefitinib $1 \mu \mathrm{M}$ for $1 \mathrm{~h}$ were incubated with $1.5,3.5,6.25$, or $12.5 \mu \mathrm{M}$ cisplatin for $24 \mathrm{~h}$ followed by immunoblot analysis of whole cell lysates (Fig. 5a, b). PARP cleavage was assessed as a marker of apoptosis while pERK and pAKT were evaluated as indicators or pro-survival/anti-apoptotic signaling. In Cal27 cells, treatment with cisplatin alone did not cause significant PARP cleavage. Treatment with gefitinib alone caused an increase in PARP cleavage, which

\section{SCC25 Cells}

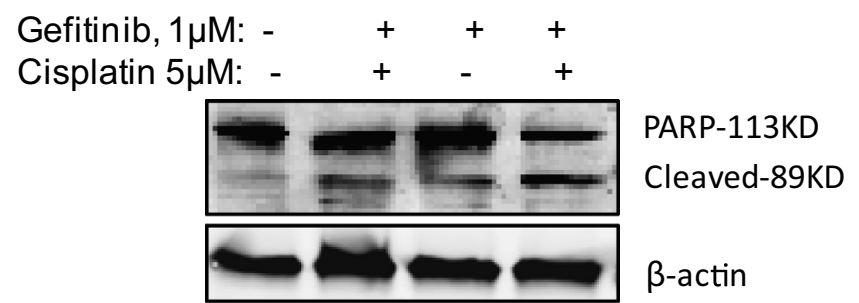

D

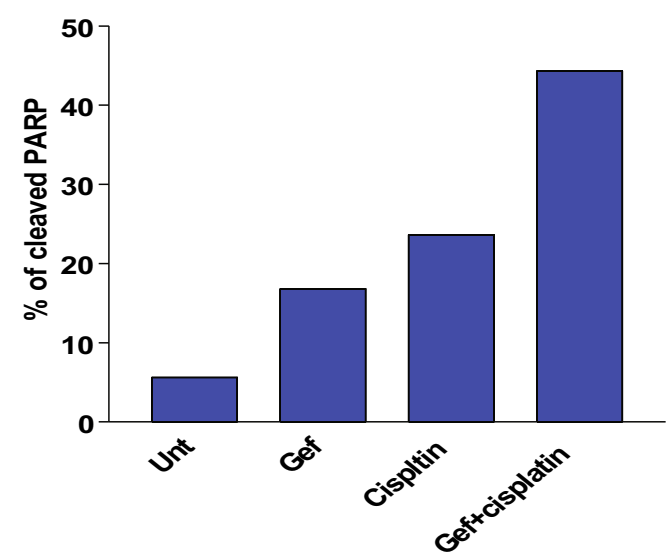

with the indicated antibodies. $\beta$-Actin was used to indicate equal protein loading. b, d Summary of densitometric analysis of cleaved PARP as a percentage of total PARP. OSCC oral cavity squamous cell carcinoma, PARP poly ADP ribose polymerase 
was augmented in a dose-dependent fashion by cisplatin. Cisplatin treatment induced a dose-dependent increase in ERK phosphorylation; this effect was inhibited by cotreatment with gefitinib.

SCC25 cells were similarly incubated with cisplatin $5 \mu \mathrm{M}$ and/or gefitinib $1 \mu \mathrm{M}$ followed by immunoblot analysis of whole cell lysates (Fig. 5c, d). Gefitinib alone and cisplatin alone caused small increases in PARP cleavage; combined gefitinib and cisplatin resulted in substantially greater PARP cleavage than either drug alone.

\subsection{Gefitinib Enhances the Apoptotic Effect of Cisplatin on OSCC Cell Lines}

Induction of apoptosis in Cal27, OSC19, and SCC25 cells was examined by flow cytometry. Cells were incubated
Fig. 6 Impact of cisplatin and gefitinib co-treatment on apoptosis in OSCC. Cal27, OSC19, and SCC25 cells were treated with gefitinib $1 \mu \mathrm{M}$, cisplatin $5 \mu \mathrm{M}$, or both for $72 \mathrm{~h}$. Cells were collected, treated with annexin V-FITC and PI, and subjected to flow cytometry as described in the text. a Representative flow cytometry results for Cal27 cells. The percentages of early and late apoptotic cells are indicated in the lower right and upper right corners, respectively. b Summary of three independent experiments showing relative percentages of apoptotic cells (early and late) for gefitinib, cisplatin, and the combination in the indicated cell lines. Data are shown as mean \pm standard error of the mean (SEM). ${ }^{*} p<0.05$ compared with control untreated cells. OSCC oral cavity squamous cell carcinoma

\section{A}

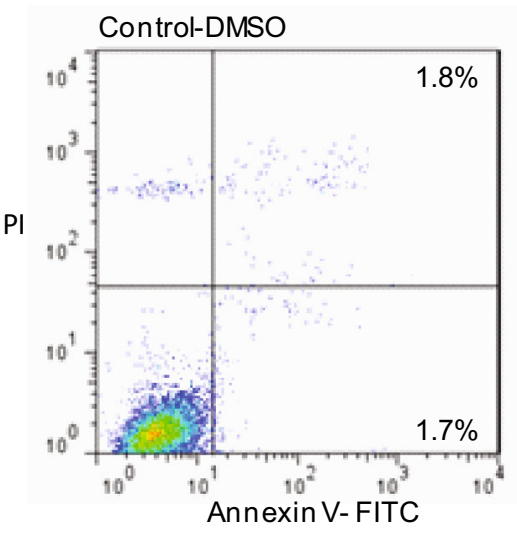

Cisplatin, $5 \mu \mathrm{M}$

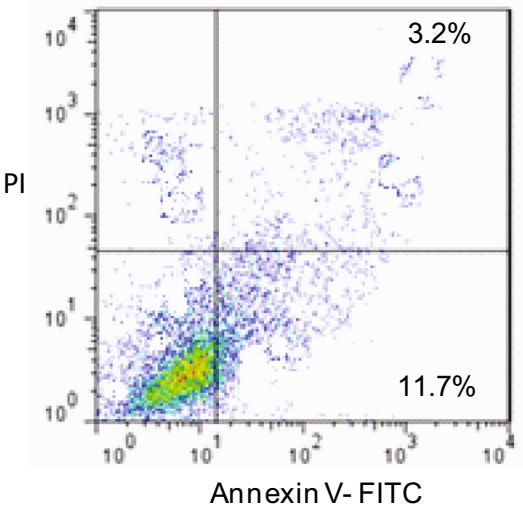

Cisplatin, $5 \mu \mathrm{M}+\mathrm{Gefitinib}, 1 \mu \mathrm{M}$

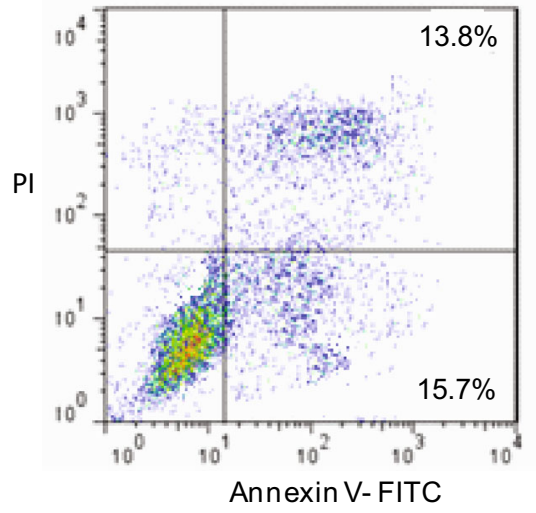

B

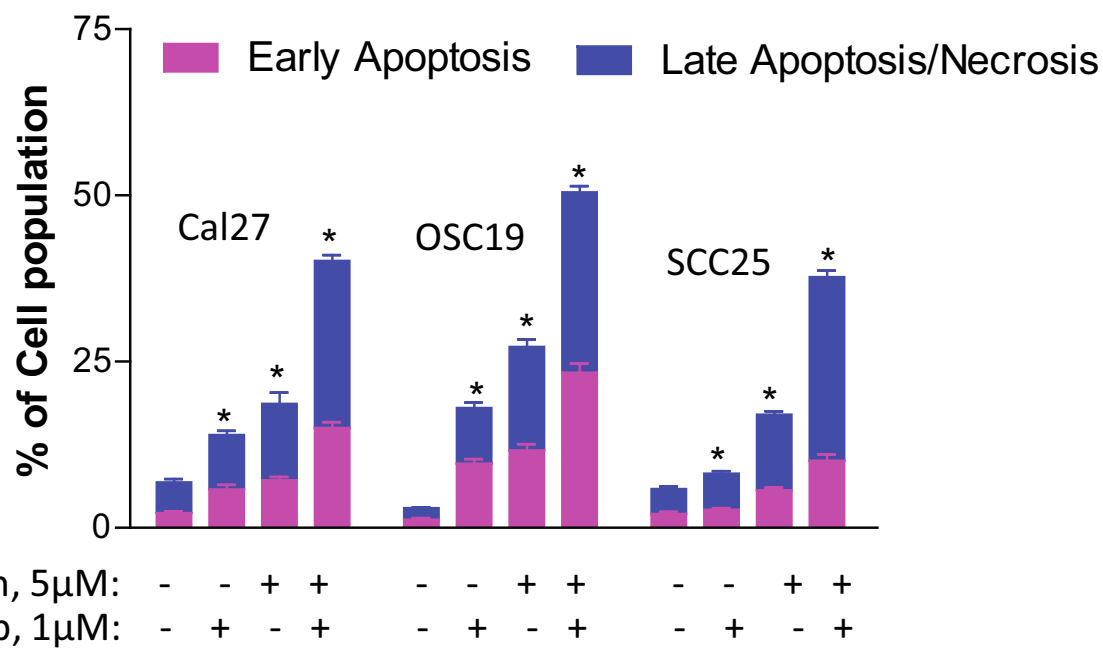


Table 1 Apoptotic and necrotic cell populations after gefitinib and cisplatin treatment of OSCC

\begin{tabular}{|c|c|c|c|c|c|c|c|c|c|c|c|c|}
\hline \multirow[t]{3}{*}{ Apoptosis } & \multicolumn{4}{|c|}{$\mathrm{Ca} 27$} & \multicolumn{4}{|c|}{ OSC19 } & \multicolumn{4}{|c|}{ SCC25 } \\
\hline & \multicolumn{2}{|c|}{ Early } & \multicolumn{2}{|l|}{ Late } & \multicolumn{2}{|c|}{ Early } & \multicolumn{2}{|l|}{ Late } & \multicolumn{2}{|c|}{ Early } & \multicolumn{2}{|l|}{ Late } \\
\hline & $M$ & SEM & $M$ & SEM & $M$ & SEM & $M$ & SEM & $M$ & SEM & $M$ & SE \\
\hline Untreated & 2.2 & 0.3 & 4.6 & 0.5 & 1.2 & 0.2 & 1.8 & 0.1 & 2.1 & 0.3 & 3.7 & 0.4 \\
\hline Gefitinib $1 \mu \mathrm{M}$ & 5.8 & 0.7 & 8.2 & 0.7 & 9.7 & 0.7 & 8.4 & 0.8 & 2.7 & 0.3 & 5.5 & 0.4 \\
\hline Cisplatin $5 \mu \mathrm{M}$ & 7.1 & 0.5 & 11.5 & 1.7 & 11.7 & 0.9 & 15.6 & 1.1 & 2.6 & 0.3 & 7.4 & 0.3 \\
\hline Gefitinib + cisplatin & 15.0 & 0.9 & 25.1 & 0.8 & 23.4 & 1.4 & 27.1 & 0.9 & 3.7 & 0.3 & 21.0 & 1.8 \\
\hline
\end{tabular}

$M$ mean, $O S C C$ oral cavity squamous cell carcinoma, SEM standard error of the mean with cisplatin $5 \mu \mathrm{M}$ either alone or in combination with gefitinib $1 \mu \mathrm{M}$ for $72 \mathrm{~h}$ and subsequently processed for flow cytometry using annexin V-FITC and PI as described in the Methods section. Figure 6a demonstrates the cell populations in early (lower right quadrant) and late (upper right quadrant) apoptosis. The results are summarized in Table 1 and Fig. 6b. The apoptotic cell population averaged 7,3 , and $6 \%$ in untreated Cal27, OSC19, and SCC25 cells, respectively. This population increased to 14,20 , and $9 \%(p<0.05)$, respectively, upon gefitinib treatment and 19,28 , and $17 \%(p<0.05)$, respectively, upon cisplatin treatment. Combined treatment with both cisplatin and gefitinib resulted in apoptosis in 40, 43, and $32 \%$ $(p<0.05)$, respectively.

\section{Discussion}

Targeted therapies using small molecules that inhibit key signaling pathways involved in tumor growth and proliferation have shown promising results against various malignant tumors in preclinical and clinical studies [28, 29]. Combining these targeted therapies to increase efficacy and reduce toxicity is an emerging therapeutic strategy [30]. One such approach is the combination of cisplatin and an EGFR inhibitor [31]. In the present study, the ability of the EGFR-TKI gefitinib to enhance the apoptotic action of cisplatin was evaluated in vitro in the OSCC cell lines Cal27, OSC19, and SCC25. The results demonstrate that, at a concentration that inhibits basal EGFR activity but does not significantly impact cell growth, gefitinib treatment significantly enhances the proapoptotic and anti-proliferative effects of cisplatin.

Compared with cisplatin alone, the combination of gefitinib and cisplatin led to significantly reduced cell numbers and colony formation in all three OSCC cell lines. This coincided with decreased ERK and AKT signaling, increased PARP cleavage, and increased apoptosis. These effects appeared to be synergistic. Treatment with cisplatin alone resulted in ERK activation in Cal27 cells, which could contribute to resistance to its growth inhibitory effect. This cisplatin-induced ERK activation was abolished by the addition of gefitinib $1 \mu \mathrm{M}$, a concentration that did not, by itself, cause growth inhibition. Thus, the increase in ERK signaling in response to cisplatin treatment may be EGFR-dependent.

These results are in agreement with numerous studies demonstrating that gefitinib inhibits cancer cell growth by mechanisms involving the inhibition of EGFR and its downstream ERK and AKT pathways [32, 33]. Consistent with the present findings, chemo-sensitizing effects of gefitinib have been reported in drug-resistant breast cancer cell lines [19, 34-37].

In this study, the expression level of EGFR in Cal27, OSC19, and SCC25 cells was high. No correlation was noted between EGFR expression level and sensitivity to gefitinib or synergism between gefitinib and cisplatin. Prior efforts to establish correlation between EGFR expression levels and the response to cetuximab or EGFR-TKI in upper aero-digestive tract squamous cell carcinoma (SCC) have been inconsistent and thus inconclusive [38]. In vitro work in head and neck SCC (HNSCC) suggests that high EGFR expression may correlate with response to EGFRTKI [39] and high EGFR copy number has also been reported to correlate with erlotinib response in HNSCC patients [24]. On the other hand, a clinical trial of erlotinib in HNSCC showed that EGFR protein levels are not indicative of erlotinib response [40]. Further, a clinical study of combining gefitinib with cisplatin and radiation therapy failed to uncover EGFR protein expression as a predictive biomarker for gefitinib responsiveness [31, 41]. Finally, in vitro studies with erlotinib or cetuximab (a monoclonal antibody against the EGFR) showed that the total levels of EGFR protein correlate with EGFR inhibitor sensitivity; in this study, EGFR was downregulated in cetuximab- and erlotinib-resistant cells [18, 42].

EGFR-activating mutations had been established as a predictive biomarker to EGFR-TKI response, particularly in non-small-cell lung cancer (NSCLC), where the inframe deletion of exon 19 and the L858R substitution in exon 21 account for $>90 \%$ of the drug-sensitive mutations. The mutations of the TK domain affect amino acids near 
the ATP-binding pocket that interfere with binding of gefitinib, and thus can be used a predictor of TKI response [43]. However, the incidence of such EGFR mutations are noticeably less common in OSCC, ranging from $0-8 \%$ depending on the ethnicity and geographical distribution, and these mutations appear different from NSCLC as it involves exon 20 more frequently [44, 45]. Therefore, EGFR mutation in OSCC does not carry the significance that it does in NSCLC [46]. Moreover, EGFR-TKI responders have been noted in some HNSCCs in the absence of EGFR mutation [24]. A phase II study of gefitinib $500 \mathrm{mg}$ daily in combination with cisplatin in Asian patients with locally advanced HNSCC failed to identify predictive biomarkers of favorable outcome with the addition of gefitinib [42, 43, 47, 48]. EGFR FISH, protein expression, and mutational status did not predict for response or survival outcome of patients [24, 49, 50]. It is clear that biomarkers predictive of response to gefitinib in HNSCC are not identical to mutations affecting the EGFR gene or its products as in NSCLC [51].

Due to the complexity of factors involved in the EGFR signaling and cross talk it should be emphasized that there are limitations involved in this type of in vitro work utilizing cancer cell lines as these cell lines may harbor genetic mutations or other genetic anomalies that could alter the experimental outcomes. For example, it was reported that the SCC25 cell line harbors a deletion mutation in cyclin dependent kinase, Cdk1, and Cal27 cells are known to harbor a nonsense mutation in SMAD4, which modulates the transforming growth factor (TGF) signaling pathway and cell growth [46, 52, 53]. Nevertheless, these cell lines are considered reliable investigative models in the search for novel and targeted therapies to treat head and neck cancer.

In clinical studies of HNSCC, hepatocellular carcinoma, and NSCLC, addition of EGFR-TKIs showed no survival benefit over placebo when combined with cisplatin $[54,55]$. Moreover, gefitinib did not improve time to progression or objective tumor response over chemotherapy alone. In a recent study of advanced HNSCC requiring postoperative chemoradiation therapy, patients were randomized to receive gefitinib or placebo in addition to cytotoxic chemotherapy. In the overall cohort, addition of gefitinib was not beneficial, but when the population was categorized according to the expression of the insulin-like growth factor-1 receptor (IGF1R), addition of gefitinib was noted to produce a statistically significant improvement in survival in patients with tumors expressing high IGF1R levels. Given the present results, it is possible that high IGF1R expression identified tumors that would benefit from gefitinib enhancement of cisplatin cytotoxicity, while in tumors with low IGF1R expression this enhancement did not occur, resulting in a lack of perceived benefit in the overall study cohort [56].

Identifying tumors in which cisplatin treatment efficacy is improved with gefitinib co-treatment could be important not only for improving cancer survival, but also for toxicity reduction if the same tumor response can be achieved with lower doses of cisplatin. This could have substantial importance in patients with OSCC, who are often malnourished and unable to tolerate maximal doses of cytotoxic drugs.

\section{Conclusion}

The EGFR-TKI gefitinib sensitizes OSCC Cal27, OSC19, and SCC25 cells to the growth inhibitory and pro-apoptotic effects of cisplatin. These in vitro observations demonstrate the need for a more thorough understanding of the molecular crosstalk in HNSCC. If a similar effect is noted in vivo, it is possible that, in appropriate settings, cotreatment with gefitinib could enhance cisplatin effects or reduce required cisplatin doses and thus toxicity. It will therefore be crucial to identify human tumors in which this effect can be observed and to find a predictor of this effect.

\section{Compliance with Ethical Standards}

Conflict of interest Ashraf Khalil declares no potential conflicts of interest; Mark J. Jameson declares no potential conflicts of interest.

Grant support The study was supported by NIH Grant K08DE019477 and American Head and Neck Society pilot Grant to M. J. Jameson.

Open Access This article is distributed under the terms of the Creative Commons Attribution-NonCommercial 4.0 International License (http://creativecommons.org/licenses/by-nc/4.0/), which permits any noncommercial use, distribution, and reproduction in any medium, provided you give appropriate credit to the original author(s) and the source, provide a link to the Creative Commons license, and indicate if changes were made.

\section{References}

1. Hu G, et al. Expression of epidermal growth factor receptor and human papillomavirus E6/E7 proteins in cervical carcinoma cells. J Natl Cancer Inst. 1997;89(17):1271-6.

2. Rubin Grandis J, et al. Levels of TGF-alpha and EGFR protein in head and neck squamous cell carcinoma and patient survival. J Natl Cancer Inst. 1998;90(11):824-32.

3. Lagha A, et al. Systemic therapy in the management of metastatic or advanced salivary gland cancers. Head Neck Oncol. 2012;4:19.

4. Hiraishi Y, et al. Immunohistochemical expression of EGFR and p-EGFR in oral squamous cell carcinomas. Pathol Oncol Res. 2006;12(2):87-91. 
5. Huang SM, Harari PM. Epidermal growth factor receptor inhibition in cancer therapy: biology, rationale and preliminary clinical results. Invest New Drugs. 1999;17(3):259-69.

6. Saeki T, et al. Association of epidermal growth factor-related peptides and type I receptor tyrosine kinase receptors with prognosis of human colorectal carcinomas. Jpn J Clin Oncol. 1995;25(6):240-9.

7. Caraglia M, et al. EGF-R small inhibitors and anti-EGF-R antibodies: advantages and limits of a new avenue in anticancer therapy. Recent Pat Anticancer Drug Discov. 2006;1(2):209-22.

8. Fukuoka M, et al. Multi-institutional randomized phase II trial of gefitinib for previously treated patients with advanced non-smallcell lung cancer (The IDEAL 1 Trial) [corrected]. J Clin Oncol. 2003;21(12):2237-46.

9. Gibbs JB. Anticancer drug targets: growth factors and growth factor signaling. J Clin Invest. 2000;105(1):9-13.

10. Mendelsohn J, Baselga J. Status of epidermal growth factor receptor antagonists in the biology and treatment of cancer. J Clin Oncol. 2003;21(14):2787-99.

11. Traxler P. Tyrosine kinases as targets in cancer therapy-successes and failures. Expert Opin Ther Targets. 2003;7(2):215-34.

12. Jackman DM, et al. A phase I trial of high dose gefitinib for patients with leptomeningeal metastases from non-small cell lung cancer. Oncotarget. 2015;6(6):4527-36.

13. Ono M, et al. Sensitivity to gefitinib (Iressa, ZD1839) in nonsmall cell lung cancer cell lines correlates with dependence on the epidermal growth factor (EGF) receptor/extracellular signal-regulated kinase 1/2 and EGF receptor/Akt pathway for proliferation. Mol Cancer Ther. 2004;3(4):465-72.

14. Herbst RS, Fukuoka M, Baselga J. Gefitinib—a novel targeted approach to treating cancer. Nat Rev Cancer. 2004;4(12):956-65.

15. Ono M, Kuwano M. Molecular mechanisms of epidermal growth factor receptor (EGFR) activation and response to gefitinib and other EGFR-targeting drugs. Clin Cancer Res. 2006;12(24):7242-51.

16. Von Pawel J. Gefitinib (Iressa, ZD1839): a novel targeted approach for the treatment of solid tumors. Bull Cancer. 2004;91(5):E70-6.

17. Han SW, et al. Predictive and prognostic impact of epidermal growth factor receptor mutation in non-small-cell lung cancer patients treated with gefitinib. J Clin Oncol. 2005;23(11):2493-501.

18. Roengvoraphoj M, et al. Epidermal growth factor receptor tyrosine kinase inhibitors as initial therapy for non-small cell lung cancer: focus on epidermal growth factor receptor mutation testing and mutation-positive patients. Cancer Treat Rev. 2013;39(8):839-50.

19. Dasari S, Tchounwou PB. Cisplatin in cancer therapy: molecular mechanisms of action. Eur J Pharmacol. 2014;740:364-78.

20. Michaelis M, et al. Cisplatin-resistant neuroblastoma cells express enhanced levels of epidermal growth factor receptor (EGFR) and are sensitive to treatment with EGFR-specific toxins. Clin Cancer Res. 2008;14(20):6531-7.

21. Mendelsohn J. The epidermal growth factor receptor as a target for cancer therapy. Endocr Relat Cancer. 2001;8(1):3-9.

22. Bourhis J. New approaches to enhance chemotherapy in SCCHN. Ann Oncol. 2005;16(Suppl 6):vi20-4.

23. Pendleton KP, Grandis JR Cisplatin-based chemotherapy options for recurrent and/or metastatic squamous cell cancer of the head and neck. Clin Med Insights Ther. 2013;2013(5). doi:10.4137/ CMT.S10409.

24. Tan EH, et al. Gefitinib, cisplatin, and concurrent radiotherapy for locally advanced head and neck cancer: EGFR FISH, protein expression, and mutational status are not predictive biomarkers. Ann Oncol. 2012;23(4):1010-6.
25. Khalil AA, Jameson MJ. Sodium orthovanadate inhibits proliferation and triggers apoptosis in oral squamous cell carcinoma in vitro. Biochem (Mosc). 2017;82(2):149-55.

26. Hiraishi Y, et al. EGFR inhibitor enhances cisplatin sensitivity of oral squamous cell carcinoma cell lines. Pathol Oncol Res. 2008;14(1):39-43.

27. Sooriyaarachchi M, Narendran A, Gailer J. Comparative hydrolysis and plasma protein binding of cis-platin and carboplatin in human plasma in vitro. Metallomics. 2011;3(1):49-55.

28. Jedlinski A, et al. EGFR status and EGFR ligand expression influence the treatment response of head and neck cancer cell lines. J Oral Pathol Med. 2013;42(1):26-36.

29. Wang X, Martindale JL, Holbrook NJ. Requirement for ERK activation in cisplatin-induced apoptosis. J Biol Chem. 2000;275(50):39435-43.

30. Takabatake D, et al. Tumor inhibitory effect of gefitinib (ZD1839, Iressa) and taxane combination therapy in EGFRoverexpressing breast cancer cell lines (MCF7/ADR, MDA-MB231). Int J Cancer. 2007;120(1):181-8.

31. Boeckx C, et al. Anti-epidermal growth factor receptor therapy in head and neck squamous cell carcinoma: focus on potential molecular mechanisms of drug resistance. Oncologist. 2013;18(7):850-64.

32. Jorissen RN, et al. Epidermal growth factor receptor: mechanisms of activation and signalling. Exp Cell Res. 2003;284(1):31-53.

33. Kjaer I, et al. Cetuximab resistance in squamous carcinomas of the upper aerodigestive tract is driven by receptor tyrosine kinase plasticity: potential for $\mathrm{mAb}$ mixtures. Mol Cancer Ther. 2016;15(7):1614-26.

34. Cappuzzo F, et al. Prospective study of gefitinib in epidermal growth factor receptor fluorescence in situ hybridization-positive/ phospho-Akt-positive or never smoker patients with advanced non-small-cell lung cancer: the ONCOBELL trial. J Clin Oncol. 2007;25(16):2248-55.

35. Anuradha V, et al. Palliative chemotherapy in head and neck squamous cell cancer-what is best in Indian population? A time without symptoms, treatment toxicity score based study. Indian J Med Paediatr Oncol. 2013;34(1):11-5.

36. Benhar M, Engelberg D, Levitzki A. Cisplatin-induced activation of the EGF receptor. Oncogene. 2002;21(57):8723-31.

37. Clark JI, et al. Phase I pilot study of oxaliplatin, infusional 5-FU, and cetuximab in recurrent or metastatic head and neck cancer. Med Oncol. 2013;30(1):358.

38. Sequist LV, et al. Molecular predictors of response to epidermal growth factor receptor antagonists in non-small-cell lung cancer. J Clin Oncol. 2007;25(5):587-95.

39. Soulieres D, et al. Multicenter phase II study of erlotinib, an oral epidermal growth factor receptor tyrosine kinase inhibitor, in patients with recurrent or metastatic squamous cell cancer of the head and neck. J Clin Oncol. 2004;22(1):77-85.

40. Quesnelle KM, et al. Preclinical modeling of EGFR inhibitor resistance in head and neck cancer. Cancer Biol Ther. 2012;13(10):935-45.

41. Zhan $P$, et al. Prognostic value of vascular endothelial growth factor expression in patients with lung cancer: a systematic review with meta-analysis. J Thorac Oncol. 2009;4(9):1094-103.

42. Thariat J, et al. Epidermal growth factor receptor protein detection in head and neck cancer patients: a many-faceted picture. Clin Cancer Res. 2012;18(5):1313-22.

43. Cohen EE, et al. Response of some head and neck cancers to epidermal growth factor receptor tyrosine kinase inhibitors may be linked to mutation of ERBB2 rather than EGFR. Clin Cancer Res. 2005;11(22):8105-8.

44. Yoshino T, et al. Platinum-based chemotherapy plus cetuximab for the first-line treatment of Japanese patients with recurrent and/ 
or metastatic squamous cell carcinoma of the head and neck: results of a phase II trial. Jpn J Clin Oncol. 2013;43(5):524-31.

45. Cappuzzo F, et al. Clinical experience with gefitinib: an update. Crit Rev Oncol Hematol. 2006;58(1):31-45.

46. Tsui IF, Garnis C. Integrative molecular characterization of head and neck cancer cell model genomes. Head Neck. 2010;32(9):1143-60.

47. Cohen EE, et al. Phase II trial of gefitinib $250 \mathrm{mg}$ daily in patients with recurrent and/or metastatic squamous cell carcinoma of the head and neck. Clin Cancer Res. 2005;11(23):8418-24.

48. Fukuoka M, et al. Biomarker analyses and final overall survival results from a phase III, randomized, open-label, first-line study of gefitinib versus carboplatin/paclitaxel in clinically selected patients with advanced non-small-cell lung cancer in Asia (IPASS). J Clin Oncol. 2011;29(21):2866-74.

49. Zhu AX, et al. Phase 2 study of cetuximab in patients with advanced hepatocellular carcinoma. Cancer. 2007;110(3):581-9.

50. Martins RG, et al. Cisplatin and radiotherapy with or without erlotinib in locally advanced squamous cell carcinoma of the head and neck: a randomized phase II trial. J Clin Oncol. 2013;31(11):1415-21.
51. Echarri MJ, Lopez-Martin A, Hitt R. Targeted therapy in locally advanced and recurrent/metastatic head and neck squamous cell carcinoma (LA-R/M HNSCC). Cancers (Basel). 2016;8(3).

52. Dahler AL, et al. Keratinocyte growth arrest is associated with activation of a transcriptional repressor element in the human cdk1 promoter. J Cell Physiol. 1998;177(3):474-82.

53. Qiu W, et al. Disruption of transforming growth factor beta-Smad signaling pathway in head and neck squamous cell carcinoma as evidenced by mutations of SMAD2 and SMAD4. Cancer Lett. 2007;245(1-2):163-70.

54. Voiculescu M, et al. Chemotherapies and targeted therapies in advanced hepatocellular carcinoma: from laboratory to clinic. J Gastrointestin Liver Dis. 2008;17(3):315-22.

55. Wu YL, et al. Phase III, randomized, open-label, first-line study in Asia of gefitinib versus carboplatin/paclitaxel in clinically selected patients with advanced non-small-cell lung cancer: evaluation of patients recruited from mainland China. Asia Pac J Clin Oncol. 2012;8(3):232-43.

56. Thariat $\mathrm{J}$, et al. Contrasted outcomes to gefitinib on tumoral IGF1R expression in head and neck cancer patients receiving postoperative chemoradiation (GORTEC trial 2004-02). Clin Cancer Res. 2012;18(18):5123-33. 